\title{
Urine and the laboratory diagnosis of Chlamydia trachomatis in males
}

\author{
T-W Kok, L E Payne, S E Bailey, R G Waddell
}

\begin{abstract}
Objective-To determine whether the use of urine samples from male patients can replace urethral swabs for the rapid detection of Chlamydia trachomatis by the Pharmacia EIA.

Setting-The STD clinic, Adelaide, South Australia.

Patients-There were two separate groups of male patients. Group A (398) patients provided urethral specimens for the EIA and culture tests. The patients in Group B (356) provided an urethral swab and a urine sample for the EIA test.

Methods-The urine samples and urethral swabs were tested for the presence of $C$ trachomatis by the Pharmacia Chlamydia EIA. In addition, the urethral swabs from Group A patients were cultured for the organism by standard cell cultures. The infected cell cultures were identified by an immunofluorescence test using a FITC-monoclonal antibody to $C$ trachomatis (Kallestad).

Results-When the EIA was validated against culture, it showed a sensitivity of $100 \%$ and a specificity of $95 \%$ with the urethral swabs from Group A patients. The urine specimens were positive in $24 \%$ of those patients who yielded a positive EIA result in the urethral swabs.

Conclusions-Although the EIA test on urethral swabs showed high sensitivity and specificity when validated against culture, our results showed that the use of urine samples cannot replace urethral swabs for the laboratory diagnosis of this sexually transmitted disease.
\end{abstract}

(Genitourin Med 1993;69:51-53)

The traditionally accepted specimen for the laboratory diagnosis of genital Chlamydia trachomatis infection is either a cervical swab from female patients or a urethral swab from male patients. Since the initial report by Adger and colleagues ${ }^{1}$ on the use of urine samples from male patients for the diagnosis of this sexually transmitted disease (STD), this less invasive sampling method has attracted much interest. ${ }^{1-4}$ These reports compared the use of urine samples in one test system (for example enzyme immunoassay (EIA) or immunofluorescence) with the corresponding urethral swab tested in another system, such as in cell cultures. The sensitivities of these urine tests ranged from $38 \% 2$ to $88 \%, 5$ when compared with the urethral specimen.
In order to assess the efficiency of using urine samples as a possible replacement for urethral swab to diagnose this infection in male patients, we tested urine and corresponding urethral samples from 356 male patients. The corresponding urine and urethral specimens were tested simultaneously in the same rapid enzyme immunoassay with a confirmatory blocking antibody test. This was done to enable a direct comparison of the performance of the assay with the two sampling methods. The EIA was first validated with a different group of male urethral specimens. Our results showed that the use of urine samples cannot replace urethral swabs for the laboratory diagnosis of this STD.

\section{Methods}

Patients

Patients were those attending the Central STD Clinic in Adelaide, South Australia. This clinic is the only STD clinic in the state and each year diagnoses about $50 \%$ of male genital chlamydia infections notified to the State Health Commission. There were two separate groups of male patients in this study. The patients in Group A attended the clinic between 1 June 1990 and 30 November 1990 and were selected on their likelihood of having a chlamydial infection according to one or more of the following criteria: [1] contact with an STD, [2] symptoms consistent with an STD, [3] > 4 polymorphs per high power field on urethral smear. Group A consisted of 398 patients (average age 29.4 years) who provided urethral specimens for EIA and culture. No urine samples were obtained from the patients in Group A.

Patients in Group B consisted of 356 males (average age 29.2 years) who presented to the clinic between 1 September 1991 and 4 November 1991 who were examined and screened for STD. They represented $59 \%$ of all male patients seen during the period.

A standardised sexual history was collected from all patients and the time of last voiding (LPU) to the nearest $\frac{1}{4}$ hour was documented. LPU information was not available in $25.6 \%$ of patients included in Group B.

\section{Urethral specimens}

In Group A three urethral specimens were collected from each patient. The first swab was cultured for gonorrhoea and used to make a Gram-stained smear which was examined for the presence of polymorphs. The second and third swabs were used for chlamydia 
test in the EIA and cell culture respectively.

\section{Urine specimens}

Each patient in Group B provided a urine sample after collection of two urethral swabs (for Gram stained smear, gonococcal culture and Chlamydia ELA). The median interval before collection of the urine sample and previous micturition was $21 / 2$ hour (range $0.5 \mathrm{~h}-5 \mathrm{~h}$ ). On receipt in the laboratory, $20 \mathrm{ml}$ of the urine sample was spun at $2500 \mathrm{~g} / 30 \mathrm{~min}$ at room temperature. The supernate was discarded and the pellet was resuspended in $0.5 \mathrm{ml}$ of the EIA diluent buffer (Pharmacia).

\section{Enzyme immunoassay}

The Pharmacia Chlamydia Confirmatory EIA was used according to the manufacturer's instructions. Each screen positive specimen was tested in the confirmatory EIA provided by the manufacturer.

\section{Chlamydia culture}

The cell culture method used was essentially that described by Kuo et al ${ }^{6}$. Once collected, the urethral specimens were immediately placed into a cryotube of chlamydia transport medium and frozen in liquid nitrogen for transport to the laboratory. Within one hour of receipt, the specimens were inoculated onto DEAE-dextran treated Buffalo Green Monkey (BGM) epithelial cells in 96-well microtitre plates (Nunc, Denmark). The plate was centrifuged at $1000 \mathrm{~g}(1 \mathrm{~h} / \mathrm{RT})$ and the medium was replaced before incubation at $37^{\circ} \mathrm{C}$ for 48 hours. After washing with PBS and fixation with methanol, the inoculated cell monolayers were stained with FITC-C trachomatis monoclonal antibody (Kallestad) for $30 \mathrm{~min} / 37^{\circ} \mathrm{C}$. The wells were then washed with PBS/20 min and $90 \%$ glycerol in Tris (pH 8.6) added to each well. The microtitre wells were then examined for specific staining using an immunofluorescence inverted microscope. The presence of one or more infected cells (showing intense cytoplasmic inclusion bodies) per well was considered $C$ trachomatis culture positive.

\section{Results}

\section{Validation of the EIA}

The EIA was first validated with urethral specimens from the 398 male patients in Group A. The test was compared with a standard cell culture method for $C$ trachomatis, as

Table 1 Relation between the Pharmacia Chlamydia confirmatory EIA and culture for the detection of $C$ trachomatis in 398 male urethral specimens (group $A$ )

\begin{tabular}{llcc}
\hline & \multicolumn{3}{l}{ Pharmacia EIA } \\
\cline { 2 - 4 } & Positive & Negative & Total \\
\hline Culture positive & 28 & 0 & 28 \\
Culture negative & 20 & 350 & 370 \\
Total & $48{ }^{\star}$ & 350 & 398 \\
\hline *Confirmed with blocking antibody. & & \\
EIA sensitivity 100\% (28/28), specificity $95 \%(350 / 370)$ \\
Prevalence by culture-7\% (28/398), by EIA-12\% (48/398)
\end{tabular}

described above. Table 1 relates the results of the Pharmacia Chlamydia EIA with the cell culture test for the detection of $C$ trachomatis in male urethral specimens. There were 28 specimens which were positive in both the EIA and culture tests. Thus the EIA test showed a sensitivity of $100 \%(28 / 28)$ when compared with cell culture. There were 20 specimens which were EIA positive, culture negative and this subgroup will be discussed below. The EIA specificity was 95\% (350/ 370) compared with cell culture.

\section{EIA test on urine and urethral samples}

The urine and urethral specimens from the 356 patients in Group B were tested in the Pharmacia EIA. Table 2 relates the results of the EIA test for $C$ trachomatis in urine and urethral samples. The corresponding urine and urethral specimens were tested in the same EIA test batch, to minimize intra-assay variations. There were 29 urethral specimens which yielded a positive EIA result. Thus, the urine specimens were positive in $24 \%(7 / 29)$ of those patients who yielded a positive EIA result in the urethral swabs. The prevalence in this group was $8 \%(29 / 356)$. All EIA positive specimens were confirmed with the blocking antibody test from the manufacturer. There were nine urine specimens which were positive in the EIA test. Within this group of nine, seven patients yielded a corresponding positive EIA test from their urethral swabs. The remaining two patients had a negative EIA from the urethral swab. The median time since last micturition for positive EIA (urethral swab or urine) was $2 \frac{1}{4}$ hours while that for EIA negative specimens was $2 \frac{1}{2}$ hours.

\section{Discussion}

The validation of the EIA kit showed that in a male population with a $C$ trachomatis infection prevalence of $7 \%$ (see table 1), the test sensitivity was $100 \%$ when compared with cell culture. The test specificity was $95 \%$. All screen EIA positive specimens were confirmed positive in the EIA with a different antibody (rabbit) from that used in the screening test (mouse monoclonal). The case notes were reviewed for the 20 urethral specimens which were EIA positive and culture negative. Within this subgroup of twenty patients six had presented to the clinic as contacts of chlamydia, while another eight

Table 2 Relation between the urine and urethral specimens from 356 male patients (group B) for the detection of $C$ trachomatis in the pharmacia confirmatory EIA

\begin{tabular}{llll}
\hline & \multicolumn{3}{l}{ Urine Specimens } \\
\cline { 2 - 4 } & Positive & Negative & Total \\
\hline Urethral specimen & 7 & 22 & $29^{\star}$ \\
Positive & 2 & 325 & 327 \\
Negative & $9^{\star}$ & 347 & 356 \\
\hline
\end{tabular}

*Confirmed with blocking antibody.

Urine sensitivity $24 \%(7 / 29)$ specificity $99 \%$ (325/327) Prevalence 8\% (29/356) 
had symptoms consistent with urethritis. There were six other patients who had no urethral symptoms or objective evidence of urethral inflammation and were probably false positives. This would tend to indicate that the specificity of the EIA in urethral swabs was better than $95 \%$.

Throughout the study there were no indications that the culturing of these specimens was suboptimal and all specimens were promptly placed in liquid nitrogen immediately after patient sampling. There was no detectable activity in the EIA with Staphylococcus aureus which may give rise to non-specific binding via the Fc portion of the detector antibody (data not shown). Previous reports have shown that it is not unusual for some specimens (from both male and female patients) to be EIA positive, but culture negative. ${ }^{7}$ This EIA kit was thus sufficiently sensitive and specific to be used for the detection of $C$ trachomatis in urethral specimens.

In screening for disease, the test specificity need not be $100 \%$, provided that the sensitivity is high and a confirmatory test is available to exclude the false positives obtained in the screening test. Thus a urine test with high sensitivity ( $>95 \%$ ) would provide a very useful screening test due to its convenience and patient acceptability. The EIA results in this study demonstrated a low yield $(9 / 356,2 \cdot 5 \%)$ in urine specimens. Compared with testing the urethral swab the urine test specificity was $99 \%(325 / 327)$ and the sensitivity was $24 \%$ (7/29) (see table 2). In a limited number of urine specimens, polyethylene glycol (PEG, MW 6,000) (6\%) was used as a precipitating agent for $C$ trachomatis. It was thought that this may increase the urine test sensitivity in the EIA by precipitating the extracellular organisms (including infected cells) in the urine samples. However, the results showed that the use of PEG did not increase the urine sensitivity in the EIA (data not shown).

Our results compare with those of others in which urine samples tested in different EIA kits showed sensitivities of $42 \%^{3}$ when compared with male urethral swabs and $37 \% 9$ when compared with female cervical/urethral swabs. The prevalence in their study populations were $11 \%^{3}$ and $7 \%, 9$ which is similar to that in our study population of male patients. (Locally, the STD clinic has a $C$ trachomatis prevalence of $4 \%$ in the female population).

It may be argued that the period before collection of the urine sample for EIA test and last micturition was too short for efficient detection of the organism by the EIA. However, in our study population, there was no correlation between the EIA positive results and the period before last micturition. It may also be argued that collection of the urine sample prior to urethral swab would have increased the urine test sensitivity. In this case, it is not clear whether or not urethral swab sensitivity would be adversely affected if urine was collected first. However, urine collected first would affect smears for gonorrhea and polymorph counts. Our clinical practice is to swab for gonorrhoea and chlamydia and make urethral smears before urine samples are collected and examined.

The use of urine instead of a urethral swab would have greater convenience and patient acceptability and thus provide a useful screening procedure in many health care settings. The earlier report by Paul and $\mathrm{Caul}^{2}$ suggested that a single urine sample could be used for multiple test comparisons as it does not involve the attendant problem of multiple sampling, which may decrease the amount of chlamydia organisms or antigen, especially with latter collected samples. However, their study did not propose urine as a replacement specimen for urethral swab. In order for urine specimens to replace male urethral swabs, the urine test sensitivity would need to be $95 \%$ positive, when compared with the urethral positive specimens tested in the same system. Our results and those of others ${ }^{3-5} 9$ showed that, for the laboratory diagnosis of $C$ trachomatis infection, urine specimens cannot replace male urethral specimens. However, other laboratories have reported varying sensitivities using urine specimens. ${ }^{2} 10$ This may reflect differences in assay systems. The patients in our study population may be considered at higher risk of chlamydial infection (that is, they were STD clinic clients), and the use of urine specimens from those with a lower risk of infection would provide an even lower yield of positive tests.

The authors wish to thank Pharmacia Australia for their support of this project.

1 Adger H, Shafer M-A, Sweet RL, Schachter J. Screening for Chlamydia trachomatis and Neisseria gonorrhoeae in adolescent males: Value of first-catch urine examination. Lancet 1984;ii: $944-5$.

2 Paul ID and Caul EO. Evaluation of three Chlamydia trachomatis immunoassays with an unbiased, noninvasive clinical sample. $\mathcal{F}$ Clin Microbiol 1990;28:220-2.

3 Schwebke JR, Clark AM, Pettinger MB, Nsubga P, Stamm WE. Use of a urine enzyme immonoassay as a diagnostic tool for Chlamydia trachomatis urethritis in men. $\mathcal{F}$ Clin Microbiol 1991;29:2446-9.

4 Sellors J, Mahony J, Jang D, et al. Rapid, on-site diagnosis of chlamydial urethritis in men by detection of antigens in urethral swabs and urine. $\mathcal{F}$ Clin Microbiol 1991;29: 407-9.

5 Chernesky M, Castriciano S, Sellors J, et al. Detection of Chlamydia trachomatis antigens in urine as an alternative to swabs and cultures. F Infect Dis 1990;161:124-6.

6 Kuo CC, Wang SP, Wentworth BB, Grayston JT. Primary isolation of TRIC organisms in HeLa 229 cells treated with DLAE-dextran. $\mathcal{F}$ Infect Dis 1972;125: 665-8.

7 Mahony J, Castriciano S, Sellors J, et al. Diagnosis of Chlamydia trachomatis genitial infections by cell culture and two enzyme immunoassays detecting different chlamydia antigens. F Clin Microbiol 1989;27:1934-8.

8 Tam MR, Stamm WE, Handsfield $\mathrm{HH}$, et al. Cultureindependent diagnosis of Chlamydia trachomatis using monoclonal antibodies. $N$ Engl $f$ Med 1984;310: 1146-50.

9 Sellors JW, Mahone JB, Jang D, et al. Comparison of cervical, urethral and urine specimens for the detection of Chlamydia trachomatis in women. F Infect Dis 1991; 164:205-8.

10 Hay PE, Thomas BJ, Gilchrist C, Plamer HM, Gilroy CB, Taylor-Robinson $\mathrm{D}$. The value of urine samples from men with non-gonococcal urethritis for the detection of Chlamydia trachomatis. Genitourin Med 1991;67:124-8. 